\title{
PERMUTATION MATRICES AND MATRIX EQUIVALENCE OVER A FINITE FIELD
}

\author{
GARY L. MULLEN \\ Department of Mathematics \\ The Pennsylvania State University \\ Sharon, Pennsylvania 16146
}

(Received March 21, 1980 and in revised form August 12, 1980)

ABSTRACT. Let $F=G F(q)$ denote the finite field of order $q$ and $F_{m \times n}$ the ring of m $x$ n matrices over $F$. Let $P_{n}$ be the set of all permutation matrices of order $n$ over $F$ so that $P_{n}$ is ismorphic to $S_{n}$. If $\Omega$ is a subgroup of $P_{n}$ and $A, B \in F$ mxn then $A$ is equivalent to $B$ relative to $\Omega$ if there exists $P \varepsilon P{ }_{n}$ such that $A P=B$. In sections 3 and 4 , if $\Omega=P_{n}$, formulas are given for the number of equivalence classes of a given order and for the total number of classes. In sections 5 and 6 we study two generalizations of the above definition.

KEY WORDS AND PHRASES. Permutation matrix, equivalence, automorphism, finite Gield.

AMS(MOS) SUBJECT CLASSIFICATION CODES: Primary 15A33, Secondary 12C99, $15 A 24$.

1. INTRODUCTION.

In a series of papers $[1-4,6-8]$ L. Carlitz, S. Cavior, and the author studied various forms of equivalence of functions over a finite field through the use of permutation groups acting on the field itself. In [9] the author defined two matrices $A$ and $B$ to be equivalent if $b_{i j}=\phi\left(a_{i j}\right)$ for some permutation $\phi$ of the field while in [10] $B$ was said to be equivalent to $A$ if $B=\phi(A)$ where $\phi(A)$ was computed by substitution. In the present paper we study another form of matrix equivalence over a finite field through the use of permutation matrices and the Pólya-deBruijn theory of enumeration.

Let $F=G F(q)$ denote the finite field of order $q=p^{b}, p$ is prime and $b \geq 1$ and let $F_{m \times n}$ denote the ring of $m \times n$ matrices over $F$ so that $\left|F_{m \times n}\right|=q^{m n}$. Let 
$P_{n}$ be the set of all $n \times n$ matrices over $F$ consisting entirely of zeros and ones with the property that there is exactly one 1 in each row and column. In the literature, such matrices have been called permutation matrices. It is not hard to show that $P_{n}$ is a group under matrix multiplication which is isomorphic to $S_{n}$. the symmetric group on $n$ letters and consequent1y has order $n$ ! If $P \varepsilon P$ the isomorphism can be defined as follows. If

$$
P\left[\begin{array}{l}
1 \\
\cdot \\
\dot{n}
\end{array}\right]=\left[\begin{array}{l}
\alpha_{1} \\
\dot{ }^{\prime} \\
\dot{\alpha}_{n}
\end{array}\right]
$$

then define $\phi_{P} \varepsilon S_{n}$ by $\phi_{P}(1)=\alpha_{1}(i=1, \ldots, n)$. Then $\Psi: P_{n} \rightarrow S_{n}$ defined by $\Psi(P)=\phi_{P}$ is an isomorphism.

\section{GENERAL THEORY.}

If $\Omega$ is a subgroup of $P_{n}$ we may make

DEFINITION 1. If $A, B \in F_{m x n}$ then $B$ is equivalent to $A$ relative to $\Omega$ if there exists $P \varepsilon \Omega$ such that $A P=B$.

This is an equivalence relation on $\mathrm{F}_{\operatorname{mxn}}$ so we let $\mu(A, \Omega)$ denote the order of the class of A relative to $\Omega$ and let $\lambda(\Omega)$ be the total number of classes induced by $\Omega$.

THEOREM 2.1. If $A, B \in F_{m \times n}$ then $B$ is equivalent to $A$ relative to $P_{n}$ if and only if the columns of $B$ are a permutation of the columns of $A$.

PROOF. Suppose $A P=B$ where $A=\left(a_{1 j}\right)$. In $P$ suppose that for $f=1, \ldots, n$ the 1 in column $f$ occurs in row $1_{f}$. Then AP $=\left(a_{1 f}\right) P=\left(a_{11_{j}}\right)$ so that colum $f$ of A becomes column $1_{j}$ of AP.

Conversely, suppose column $j$ of $A$ is column $I_{j}$ of $B$. Define $P$ so that in column $f$ we have a 1 in row $i_{f}$ and zeros elsewhere. Then $P \varepsilon P_{n}$ and $A P=B$ so that $A$ is equivalent to $B$.

COROLLARY 2.2. If $A, B \in F_{n \times n}$ and $B$ is equivalent to $A$ relative to $\Omega$ then $\operatorname{det}(B)= \pm \operatorname{det}(A)$.

In fact, if $A P=B$ and $P$ corresponds to $\phi_{P} \varepsilon S_{n}$ where $\phi_{P}$ is an even permutation then $\operatorname{det}(B)=\operatorname{det}(A)$ while if $\phi_{P}$ is an odd permutation then $\operatorname{det}(B)=-\operatorname{det}(A)$. 
DEFINITION 2. If $A \in F_{m \times n}$ then $P$ is an automorphism of $A$ relative to $\Omega$ if $\mathrm{P} \in \Omega$ and $\mathrm{AP}=\mathrm{A}$.

If Aut $(A, \Omega)$ denotes the set of all automorphisms of A relative to $\Omega$, then it is easy to check that $A u t(A, \Omega)$ is a group under matrix multiplication whose order will be denoted by $v(A, \Omega)$. It is easy to prove

THEOREM 2.3. If $A \in F_{m \times n}$ then for any subgroup $\Omega$ of $P_{n}$

$$
\mu(A, \Omega) \cup(A, \Omega)=|\Omega|,
$$

where $|\Omega|$ denotes the order of $\Omega$.

If $P \in P_{n}$ let $N(P, m, n, q)$ denote the number of $m \times n$ matrices $A$ over $G F(q)$ such that $A P=A$.

THEOREM 2.4. If $P$ corresponds to $\phi_{P} \varepsilon S_{n}$ and $\phi_{P}$ has $\ell(P)$ distinct cycles then $\mathrm{N}(\mathrm{P}, \mathrm{m}, \mathrm{n}, \mathrm{q})=\mathrm{q}^{\mathrm{m \ell}(\mathrm{P})}$.

PROOF. Suppose the distinct cycles of $\phi_{P}$ are $\sigma_{1}, \ldots, \sigma_{\ell(P)}$. Using Theorem 2.1 it is clear that $A P=A$ if and only if within a given cycle of $\phi_{P}$ the columns of $A$ are identical. The theorem then follows from the fact that a given column can be constructed in $\mathrm{q}^{\mathrm{m}}$ ways.

\section{CYCLIC GROUPS.}

If $\Omega=\langle$ P $\rangle$ is a cyclic group of permutation matrices where $|\Omega|=s$, let $H(t)$ denote the subgroup of $\Omega$ of order $t$ where $t \mid s$ so that $H(t)=\left\langle P^{s / t}\right\rangle$. If $P$ corresponds to $\phi_{P} \varepsilon S_{n}$ let $\ell_{t}(P)$ denote the number of cycles of $\phi_{P} s / t$ and suppose $M(t, m$, $n, q)$ denotes the number of $m \times n$ matrices $A$ over $G F(q)$ such that Aut $(A, \Omega)=H(t)$.

THEOREM 3.1. For each divisor $t$ of $s$

where $\mu(a)$ is the Mobius function.

$$
M(t, m, n, q)=\sum_{a \mid \frac{a}{t}} \mu(a) q^{m l_{a t}(P)},
$$

PROOF. By Theorem $2.4 \mathrm{q}^{\mathrm{ml}(\mathrm{P})}$ counts the number of $\mathrm{m} \times \mathrm{n}$ matrices $\mathrm{A}$ over GF( $q)$ such that Aut $(A, \Omega) \leq H(t)$. From this we subtract those for which the containment is proper. This number is given by

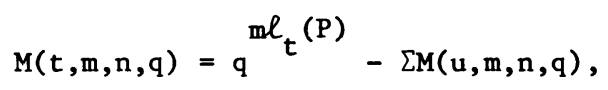

where the sum is over all $u|s, t| u$ and $t \neq u$. After applying Mobius inversion 
to (3.2) we obtain (3.1).

COROLLARY 3.2. For each divisor $t$ of $s$ there are $t M(t, m, n, q) / s$ classes of $s / t$ and

$$
\lambda(\Omega)=\frac{1}{s} \sum_{t, / s} t M(t, m, n, q) .
$$

As an illustration, suppose $q=2, m=n=3$, and

$$
P=\left[\begin{array}{lll}
0 & 1 & 0 \\
0 & 0 & 1 \\
1 & 0 & 0
\end{array}\right]
$$

so that if $\Omega=\langle\mathrm{P}\rangle$ then $|\Omega|=3$. One can easily check that $\mathrm{M}(3,3,3,2)=8$ and $M(1,3,3,2)=504$ so that there are 168 classes of order 3,8 classes of order 1 and thus from (3.3), $\lambda(\Omega)=176$.

\section{THE CASE $\Omega=P_{n}$.}

In this section we consider the group $P_{n}$ of all permutation matrices of order $n$ so that, as noted in the introduction, $P_{n}$ is isomorphic to $s_{n}$, the symmetric group on $n$ letters. We will employ the Pólya theory of enumeration to determine the number of classes induced by $P_{n}$. Suppose the permutation group $K$ acts on a et of $r$ elements. If $\pi \in k$ consider the monomial $x_{1}{ }^{b_{1}} x_{2}{ }^{b}{ }_{2} \ldots x_{r}{ }^{b}$ where for $t=1, \ldots, r b_{t}$ denotes the number of cycles of $\pi$ of length $t$. The polynomial

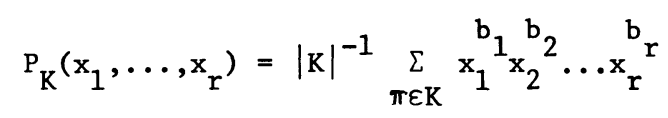

is called the cycle index of $\mathrm{K}$. It is well known [5] that $\mathrm{P}_{\mathrm{S}_{\mathrm{n}}}\left(\mathrm{x}_{1}, \ldots, \mathrm{x}_{\mathrm{n}}\right)=\Sigma\left(\mathrm{k}_{1} ! \mathrm{k}_{2} ! 2^{\mathrm{k}_{2}} \ldots \mathrm{k}_{\mathrm{n}} ! \mathrm{n}^{\mathrm{k}}\right)^{-1} \mathrm{x}_{1} \mathrm{k}_{1} \mathrm{x}_{2}{ }^{\mathrm{k}} \ldots \mathrm{x}_{\mathrm{n}}{ }^{\mathrm{k}}$ where the sum is over all $\mathrm{k}_{1}+2 \mathrm{k}_{2}+\ldots+\mathrm{nk}_{\mathrm{n}}=\mathrm{n}$.

In the Pólya theory of enumeration, let the domain $D$ be the set of $n$ columns and let the range $R$ be the set of $q^{m}$ possible column vectors so that $\left|\mathrm{R}^{\mathrm{D}}\right|=\mathrm{q}^{\mathrm{mn}}=\left|\mathrm{F}_{\mathrm{mxn}}\right|$. If $\mathrm{K}^{\dot{\prime}}$ is a permutation group acting on $\mathrm{D}$ then Pó1ya's theorem $[5, \mathrm{p} .157]$ states that the number of distinct classes is given by $\mathrm{P}_{K}(|R|, \ldots,|R|)$ so that $\lambda\left(P_{n}\right)=P_{S_{n}}\left(q^{m}, \ldots, q^{m}\right)$. It follows direct1y from Theorem 2.1 that $\lambda\left(P_{n}\right)$ is also the number of distributions of $n$ indistinguishable objects into $q^{m}$ labelled cells, or $\left({ }^{n}+q_{n}^{m}-1\right)$ so that we have proven 
Theorem 4.1. If $\lambda\left(P_{n}\right)$ is the number of classes induced by $P_{n}$ then

$$
\lambda\left(P_{n}\right)=\left(\begin{array}{c}
n+q^{m}-1 \\
n
\end{array}\right.
$$

Suppose $A \varepsilon F_{m \times n}$ has $t$ distinct columns so that we have a partition of $n$ with $t$ parts say $n=m_{1}+\ldots+m_{t}$ where each distinct column occurs $m_{i} t i m e s$. By Theorem 2.1 for each such $A$ we have $\nu\left(A, P_{n}\right)=\prod_{i=1}^{t} m_{i}:$ so that by $(2.1) \mu\left(A, P_{n}\right)=\left(m_{1}, \ldots, n, m_{t}\right)$. The number of such $A$ is the same as the number of functions from $D$ into $R$ whose range is of size $\mathrm{q}^{\mathrm{m}}$, whose domain is of size $\mathrm{n}$ and whose preimage partition has type $m_{1}+\ldots+m_{t}=n$. We may rewrite this with distinct $m^{\prime} s$ say $j_{m_{1}} m_{1}+\cdots+j_{m_{s}} m_{s}=n$ where $j_{m_{1}}+\ldots+j_{m_{s}}=t$. Then the number of such functions is $\left(q^{m^{m}}{ }_{t} h\left(j_{m_{1}}, \ldots, j_{m_{s}}\right)\right.$ where $h\left(j_{m_{1}}, \ldots, j_{m_{s}}\right)$ is the number of partitions of $n$ of type $j_{m_{1}} m_{1}+\ldots+j_{m_{s}} m_{s}=n$ and is given by Cauchy's formula

$$
h\left(j_{m_{1}}, \ldots, j_{m_{s}}\right)=n ! /\left(\left(m_{1} !\right)^{j_{m_{1}}}\left(j_{m_{1}}\right) ! \ldots\left(m_{s} !\right)^{j_{m_{s}}}\left(j_{m_{s}}\right) !\right)
$$

and $\left(q^{m}\right)_{t}=q^{m}\left(q^{m}-1\right) \ldots\left(q^{m}-t+1\right)$ is the falling factorial which assigns image values to the partition blocks. Hence we have proven

COROLLARY 4.2 . The number of classes induced by $P_{n}$ of order $\left(m_{1}, \ldots, m_{s}\right)$ is

$$
\left(\mathrm{q}_{\mathrm{t}}^{\mathrm{m}}\right)\left(\mathrm{j}_{\mathrm{m}_{1}}, \ldots, \mathrm{j}_{\mathrm{m}_{\mathrm{s}}}\right)
$$

As an illustration of the above theory suppose $q=2$ and $m=n=3$ so that we are considering the $5123 \times 3$ matrices over GF(2) under the action of the symmetric group $\mathrm{S}_{3}$. Thus from Corollary 4.2 when $\mathrm{t}=1$ we have $\mathrm{n}=3$ so that there are $\left(\begin{array}{l}8 \\ 1\end{array}\right)\left(\begin{array}{l}1 \\ 1\end{array}\right)=8$ classes of order 1 , when $t=2$ we have $n=1+2$ so that there are $\left(\begin{array}{l}8 \\ 2\end{array}\right)\left(\begin{array}{c}2 \\ 1,1\end{array}\right)=56$ classes of order 3 and when $t=3$ we have $n=1+1+1$ so that there are $\left(\begin{array}{l}8 \\ 3\end{array}\right)\left(\begin{array}{l}3 \\ 3\end{array}\right)=56$ classes of order 6 so that $\lambda\left(P_{3}\right)=120$. Moreover, from Theorem 4.1 we also see that $\lambda\left(P_{3}\right)=\left(\begin{array}{c}10 \\ 3\end{array}\right)=120$ classes.

\section{A GENERALIZATION}

In this section we generalize Definition 1 by considering a notion of matrix equivalence which is similar to the idea of weak equivalence of functions over a finite field considered by Cavior and the author in [3] and [8]. Let $P_{m}$ be the group of $\mathrm{m} \times \mathrm{m}$ permutation matrices over $\mathrm{GF}(\mathrm{q})$. If $\Omega_{1}$ is a subgroup of $P_{\mathrm{m}}$ and $\Omega_{2}$ is a subgroup of $P_{n}$ we may make 
DEFINITION 3. If $A, B \in F_{m \times n}$ then $B$ is equivalent to $A$ relative to $\Omega_{1}$ and $\Omega_{2}$ if there exist $Q \in \Omega_{1}$ and $P \varepsilon \Omega_{2}$ such that $\mathrm{QAP}=\mathrm{B}$.

Thus $P \varepsilon P_{n}$ permutes the columns of $A$ while $Q \varepsilon P_{m}$ permutes the rows of $A$ so that $\Omega_{1}$ acts as a permutation group on the range $R$ and $\Omega_{2}$ is a permutation group acting on the domain $D$. Clearly if $\Omega_{1}=\{i d$. $\}$ we obtain the previous cases considered in sections 3 and 4 . In this more general setting we will make use of the extended Pólya theory of enumeration.

THEOREM 5.1. (Polya-deBruijn) The number of classes induced by permutation groups $\Omega_{2}$ of $D$ and $\Omega_{1}$ of $R$ is

$$
\left.P_{\Omega_{2}}\left(\frac{\partial}{\partial z_{1}}, \frac{\partial}{\partial z_{2}}, \ldots\right) P_{\Omega_{1}}\left(e^{z_{1}+z_{2}+\ldots}, e^{2\left(z_{2}+z_{4}+\ldots\right)}, \ldots\right)\right|_{z_{1}=z_{2}=\ldots=0}
$$

Consider the $q^{m}$ possible column vectors of $R$ in an $m \times q^{m}$ array so that in row 1 , we have $q^{m-i+1}$ sets where in each set one element of $G F(q)$ is repeated $q^{i-1}$ times. For example, if $q=2$ and $m=3$ we list the 8 column vectors as

$$
\begin{array}{lllllllll}
0 & 1 & 0 & 1 & 0 & 1 & 0 & 1 \\
0 & 0 & 1 & 1 & 0 & 0 & 1 & 1 & . \\
0 & 0 & 0 & 0 & 1 & 1 & 1 & 1
\end{array} .
$$

Suppose now that $\Omega$ is the cyclic group of order $m$ generated by the permutation $\phi=(12 \ldots \mathrm{m})$. By letting $\Omega$ permute the rows of the $\mathrm{m} \times \mathrm{q}^{\mathrm{m}}$ array, we induce a permutation group $\Omega_{1}$ on the column vectors of the range $R$. For example, if $\phi_{Q}=(123)$ then the column vectors $\left(C_{1}, \ldots, C_{8}\right)$ of $(5.2)$ are permuted to $\left(c_{1}, c_{3}, c_{5}, c_{7}, c_{2}, c_{4}, c_{6}, c_{8}\right)$. If

$$
A=\left[\begin{array}{lll}
1 & 0 & 1 \\
1 & 1 & 0 \\
0 & 1 & 1
\end{array}\right]=\left[C_{4} C_{7} C_{6}\right]
$$

and $Q$ is the permutation matrix

$$
Q=\left[\begin{array}{lll}
0 & 0 & 1 \\
1 & 0 & 0 \\
0 & 1 & 0
\end{array}\right]
$$


then $Q A=\left[\begin{array}{lll}0 & 1 & 1 \\ 1 & 0 & 1 \\ 1 & 1 & 0\end{array}\right]=\left[C_{7} C_{6} C_{4}\right]$.

By the isomorphism defined in section $1, \phi_{Q} \varepsilon S_{3}$ corresponds to the permutation matrix $Q$. Thus by applying $\phi_{Q}$ to the rows of the $m \times q^{m}$ array, we induce a permutation on the column vectors of the range $R$. This is turn induces a permutation of the rows of $A$ which is equivalent to just permuting the rows of $A$ by using the permutation matrix Q. Hence we can permute the rows of any matrix by simply permuting the rows of the $m \times q^{m}$ array.

If $\Omega_{1}$ is the cyclic group of prime order $m$ acting on the $q^{m}$ column vectors induced by a cyclic group of prime order $m$ acting on the rows of the $m x^{m}$ array, it is not difficult to prove that

$$
\mathrm{P}_{\Omega_{1}}\left(\mathrm{x}_{1}, \ldots, \mathrm{x}_{\mathrm{q}}{ }^{\mathrm{m}}\right)=\frac{1}{\mathrm{~m}}\left(\mathrm{x}_{1}^{\mathrm{q}^{\mathrm{m}}}+(\mathrm{m}-1) \mathrm{x}_{1}^{\mathrm{q}} \mathrm{x}_{\mathrm{m}}\left(\mathrm{q}^{\mathrm{m}-\mathrm{q}) / \mathrm{m}}\right) .\right.
$$

We are now ready to prove

THEOREM 5.2. If $\Omega_{1}$ is cyclic of prime order $m$ and $\Omega_{2}$ is cyclic of order $n$ then if $\mathrm{m} / \mathrm{n}$

$$
\lambda\left(\Omega_{2}, \Omega_{1}\right)=\frac{1}{m n} \sum_{t \mid n} \phi(t)\left(q^{m n / t}+(m-1) q^{n / t}\right),
$$

while if $\mathrm{m} . \mathrm{n}$

$$
\lambda\left(\Omega_{2}, \Omega_{1}\right)=\frac{1}{m n} \sum_{\substack{t \mid n \\ t \neq k m}} \phi(t)\left(q^{m n / t}+(m-1) q^{n / t}\right)+\frac{1}{n} \sum_{\substack{t \mid n \\ t=k m}} \phi(t) q^{m n / t} .
$$

PROOF. We must evaluate (5.1) which becomes for fixed $t \mid n$

$$
\frac{\phi(t)}{m n} \frac{\partial^{n / t}}{\partial z_{t}^{n / t}} e^{q^{m}\left(z_{1}+z_{2}+\ldots\right)}+(m-1) e^{q\left(z_{1}+z_{2}+\ldots\right)} e^{\left(q^{m}-q\right)\left(z_{m}+z_{2 m}+\ldots\right)}
$$

If $t=1(5.6)$ reduces to $1 / \mathrm{mn}\left[\mathrm{q}^{\mathrm{mn}}+(\mathrm{m}-1) \mathrm{q}^{\mathrm{n}}\right]$. If $\mathrm{m} \mid \mathrm{n}$ and $\mathrm{t}>1$ is a divisor of $n$ we have $M=\phi(t) / m n\left(q^{m n / t}+(m-1) q^{n / t}\right)$ which proves (5.4) upon summing over al1 $t \mid n$. If $m \mid n$ and $1<t \neq k m$ for some positive integer $k$ the (5.6) contributes $M$ as before while if $1<t=k m$ for some $k,(5.6)$ contributes $\left(\phi(t) q^{\mathrm{mn} / \mathrm{t}}\right) / \mathrm{n}$ from which (5.5) follows.

As an illustration, suppose $\mathrm{q}=\mathrm{m}=\mathrm{n}=2$ so that we are considering the 16 
$2 \times 2$ matrices over GF(2). Let $\Omega_{1}$ be the cyclic group of order 2 acting on the two rows of the $2 \times 4$ array

$$
\begin{array}{llll}
0 & 1 & 0 & 1 \\
0 & 0 & 1 & 1
\end{array}
$$

and let $\Omega_{2}$ be the cyclic group of order 2 acting on the 2 columns of $D$. Then from (5.5) we have $\lambda\left(\Omega_{2}, \Omega_{1}\right)=5+2=7$ distinct classes which may also be easily verified by direct calculation.

\section{A FURTHER GENERALIZATION}

In this section we consider a further generalization by allowing $\Omega_{1}$ to act directly on the column vectors of $R$ rather than on the rows of the $m \times q^{m}$ array. As before suppose $\Omega_{2}$ acts on the set of $n$ columns of $D$. Thus, after a matrix is permuted by columns, it is then acted upon be a more general permutation of the column vectors of $\mathrm{R}$ rather than fust permuting the rows of the given matrix. For example, using the example from section 5 , suppose $\Omega_{1}$ is the cyclic group of order 8 generated by $\phi=(12 \ldots 8)$. Then if $\phi$ is applied to the matrix

$$
A=\left[\begin{array}{lll}
1 & 0 & 1 \\
1 & 1 & 0 \\
0 & 1 & 1
\end{array}\right]=\left[C_{4} C_{7} C_{6}\right]
$$

we obtain the matrix

$$
\left[C_{5} C_{8} C_{7}\right]=\left[\begin{array}{lll}
0 & 1 & 0 \\
0 & 1 & 1 \\
1 & 1 & 1
\end{array}\right]
$$

which cannot be obtained from A by just permuting the rows of A. Hence we have a more general setting than that considered in section 5 where equivalent matrices were obtained by simply permuting the rows and columns of the given matrix.

Suppose $\Omega_{1}$ is cyclic of order $q^{m}$ acting on the $q^{m}$ column vectors of $R$ while $\Omega_{2}$ is cyclic of order $n$ acting on the $n$ columns of $D$.

THEOREM 6.1. If $\mathrm{p} / \mathrm{n}$

$$
\lambda\left(\Omega_{2}, \Omega_{1}\right)=\frac{1}{n q^{m}} \sum_{t \mid n} \phi(t) q^{m n / t}
$$


while if $\cdot \mathrm{p} / \mathrm{n}$

$$
\lambda\left(\Omega_{2}, \Omega_{1}\right)=\frac{1}{n q^{m}}\left[\sum_{\substack{t \mid \mathrm{h} \\ t \neq k p^{i}}} \phi(t) q^{m n / t}+\sum_{\substack{t \mid n \\ t=k p^{i}}} \phi(t)\left(p^{i}-p^{i-1}+1\right) q^{m n / t}\right] .
$$

PROOF. Since $q=p^{b}$ where $p$ is a prime and $b \geq 1$ we have

$$
\begin{aligned}
& P_{\Omega_{1}}\left(x_{1}, \ldots, x_{q^{m}}\right)=\frac{1}{q^{m}} \sum_{t / q^{m}} \phi(t) x_{t}^{q^{m} / t} \\
= & \frac{-1}{q^{m}}\left[x_{1}^{p^{b m}}+\sum_{i=1}^{b m}\left(p^{i}-p^{i-1}\right) x_{p^{i}}^{b m-i}\right] .
\end{aligned}
$$

Substituting $\mathrm{P}_{\Omega_{1}}$ and $\mathrm{P}_{\Omega_{2}}$ into (5.1) we obtain for a general term with $t$ fixed

$$
\left.N=\frac{\phi(t)}{n q^{m}} \frac{\partial^{n / t}}{\partial z_{t}^{n / t}}\left[e^{p^{b m}\left(z_{1}+z_{2}+\ldots\right)}+\sum_{i=1}^{b m}\left(p^{i}-p^{i-1}\right) e^{p^{b m}\left(z^{i}+z\right.} 2 p^{i}+\ldots\right)\right]\left.\right|_{z_{i}=0}
$$

If $\mathrm{t}=1, \mathrm{~N}=\mathrm{q}^{\mathrm{mn}} /\left(\mathrm{nq}^{\mathrm{m}}\right)$ while if $\mathrm{t}^{\prime}>1$ and $\mathrm{p} / \mathrm{n}$ then $\mathrm{t} \neq \mathrm{kp}^{\mathrm{i}}$ so that

$\mathrm{N}=\left(1 / \mathrm{nq} \mathrm{m}^{\mathrm{m}}\right) \phi(\mathrm{t}) \mathrm{q}^{\mathrm{mn} / \mathrm{t}}$ from which (6.1) follows after summing over all $\mathrm{t} / \mathrm{n}$. In the case where $p \mid n$, if $t$ is a divisor of $n$ and $t \neq k p^{i}$ for some $k$ then $N$ is the same as in the above case. If $t=k p^{i}$ then $N=\left(1 / n q^{m}\right) \phi(t)\left(p^{i}-p^{i-1}+1\right) q^{m n / t}$ so that summing over all $\mathrm{t} \mid \mathrm{n}$ yields (6.2).

As an illustration, if $q=p=m=n=2$ then using (6.2) we see that $\lambda\left(\Omega_{2}, \Omega_{1}\right)=3$ so that the $162 \times 2$ matrices over $G F(2)$ are decomposed into 3 disjoint equivalence classes.

\section{REFERENCES}

1. Carlitz, L. "Invariantive theory of equations in a finite field", Trans. Amer. Math, Soc. 75 (1953), 405-427.

2. Carlitz, L. "Invariant theory of systems of equations in a finite field", J. Analyse Math. $3(1953 / 54), 382-413$.

3. Cavior, S.R. "Equivalence classes of functions over a finite field", Acta Arith. 10 (1964), 119-136.

4. Cavior, S.R. "Equivalence classes of sets of polynomials over a finite field", J. fur die Reine und Angewandte Math 225 (1967), 191-202.

5. deBruijn, N.G. Polya's theory of counting, Applied Combinatorial Mathematics (ed. E.F. Beckenbach), John Wiley \& Sons, New York, 1964. 
6. Mullen, G.L. "Equivalence classes of functions over a finite field", Acta Arith. 29 (1976), 353-358.

7. Mullen, G.L. "Equivalence classes of polynomials over fintte fields", Acta Arith. 31 (1976), 113-123.

8. Mullen, G.L. "Weak equivalence of functions over a finite field", Acta Arith. 35 (1978), 157-170.

9. Mullen, G.L. "Equivalence classes of matrices over finite fields", Lin. Alg. \& its Apps. 27 (1979), 61-68.

10. Mullen, G.L. "Equivalence classes of matrices over a finite fiedd", Internat. J. Math. \& Math. Sci. 2 (1979), 487-481. 


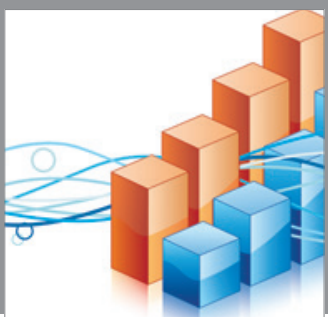

Advances in

Operations Research

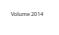

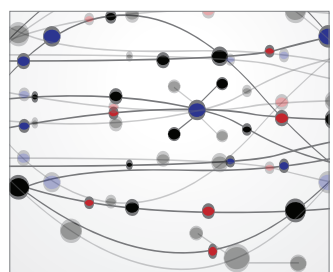

\section{The Scientific} World Journal
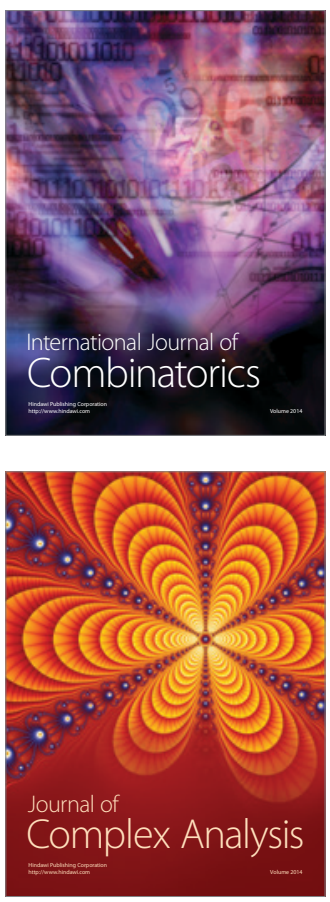

International Journal of

Mathematics and

Mathematical

Sciences
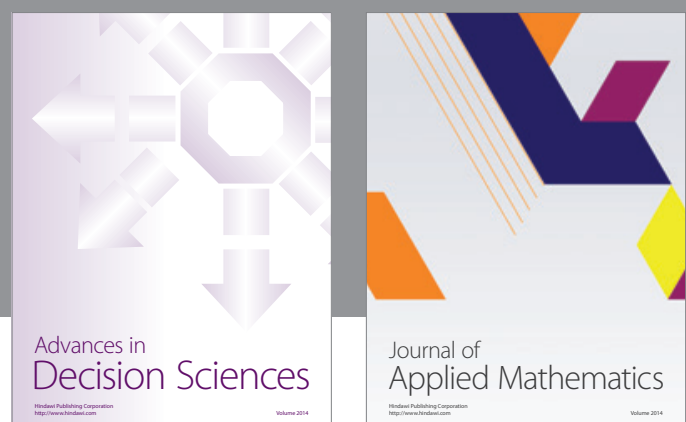

Journal of

Applied Mathematics
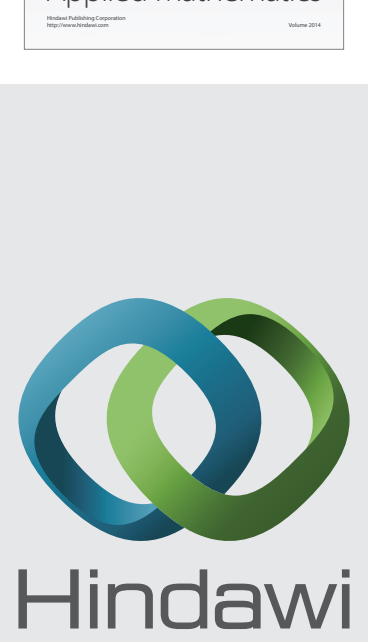

Submit your manuscripts at http://www.hindawi.com
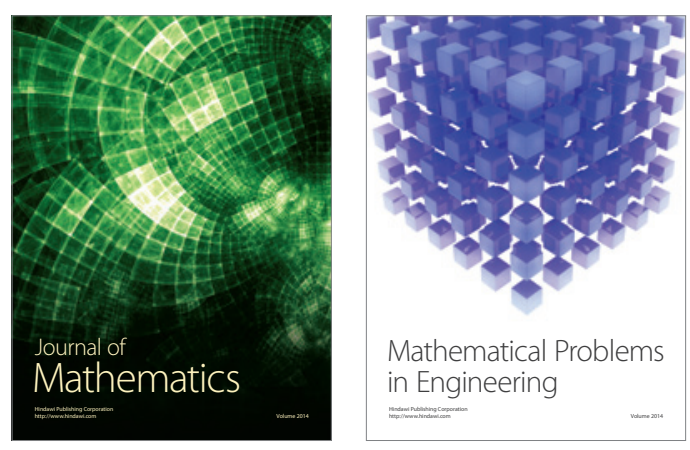

Mathematical Problems in Engineering
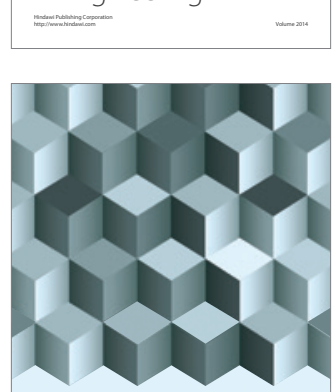

Journal of

Function Spaces
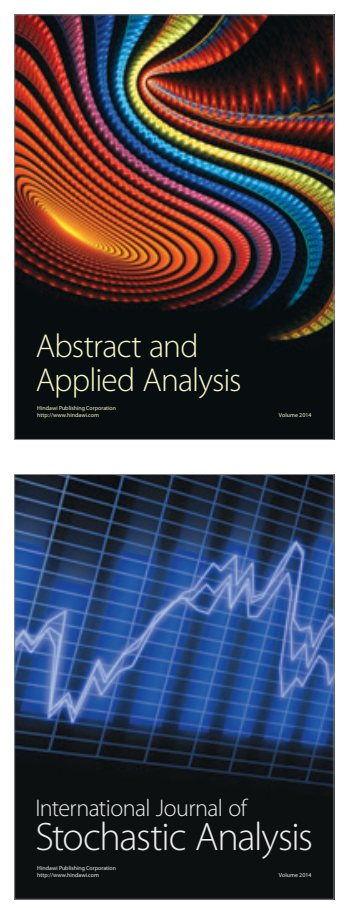

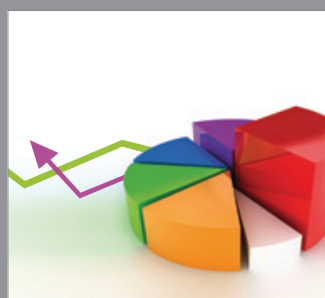

ournal of

Probability and Statistics

Promensencen
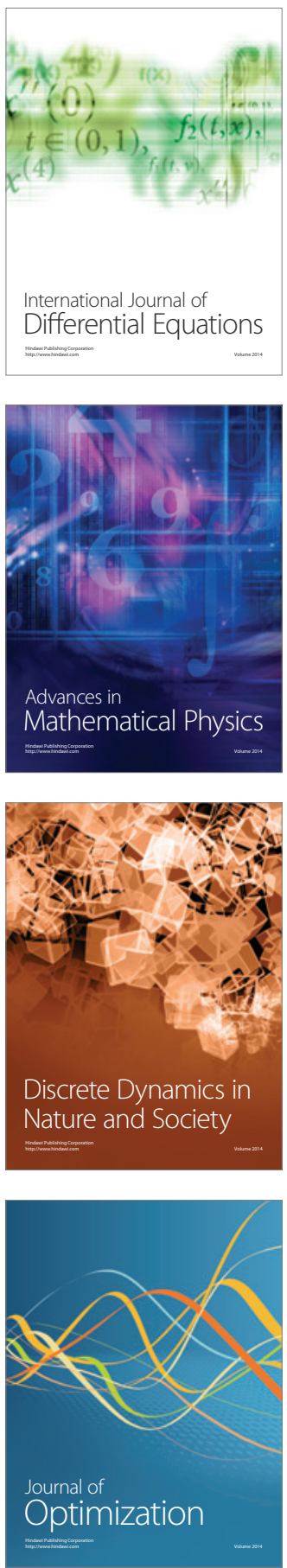